\title{
Genetic variants in AKR1B10 associate with human eating behavior
}

\author{
Kerstin Rohde ${ }^{1}$, Martin Federbusch ${ }^{2}$, Annette Horstmann ${ }^{1,2}$, Maria Keller ${ }^{1}$, Arno Villringer ${ }^{1,2,4}$, Michael Stumvoll ${ }^{1,3}$, \\ Anke Tönjes ${ }^{3}$, Peter Kovacs ${ }^{1}$ and Yvonne Böttcher ${ }^{1^{*}}$
}

\begin{abstract}
Background: The human Aldoketoreductase 1 B10 gene (AKR1B10) encodes one of the enzymes belonging to the family of aldoketoreductases and may be involved in detoxification of nutrients during digestion. Further, AKR1B10 mRNA (messenger ribonucleic acid) expression was diminished in brain regions potentially involved in the regulation of eating behavior in rats which are more sensitive to cocaine and alcohol. We hypothesized that the human AKR1B10 gene may also play a role in the regulation of human eating behavior.

Results: We investigated the effects of 5 genetic variants of AKR1B10 on human eating behavior among 548 subjects from a German self-contained population, the Sorbs, and in 350 subjects from another independent German cohort. Among the Sorbs, we observed nominal associations with disinhibition at the $5^{\prime}$ untranslated region (5' UTR) variant rs 10232478 and the intragenic variants rs 1834150 and rs782881 (all $P \leq 0.05$ ). Further, we detected a relationship of rs 1834150 and rs782881 with waist, smoking consumption (rs782881) and coffee consumption (rs1834150) (all $P \leq 0.05)$. Albeit non-significant, replication analyses revealed similar effect directions for disinhibition at rs1834150 (combined $P=0.0096$ ). Moreover, in the replication cohort we found rs 1834150 related to increased restraint scores with a similar direction as in the Sorbs (combined $P=0.0072$ ).
\end{abstract}

Conclusion: Our data suggest that genetic variants in the AKR1B10 locus may influence human eating behavior.

Keywords: Eating behavior, Association, Restraint, Disinhibition, Genetic variants

\section{Background}

The human aldoketoreductase 1B10 (AKR1B10) was identified in human hepatocellular cancer as a member of the AKR superfamily [1]. The NADP $(\mathrm{H})$-dependent (nicotinamide adenine dinucleotide phosphate) enzyme is mostly expressed in the gastrointestinal tract, particularly in the small intestine, and colon as well as adrenal gland $[1,2]$. It reduces a wide spectrum of natural or synthetic harmful components present in daily nutrients and may hence be involved in detoxification mechanisms [3]. AKR1B10 is further involved in fatty acid biosynthesis $[4,5]$ as well as the prostaglandin and retinoid acid metabolism [6-8]. It was shown that up-regulation of the mRNA or protein expression results in a tumorigenic cell proliferation [7] in several types of cancers such as liver [9], lung [10] and breast cancer [11]. Interestingly, its up-regulation was

\footnotetext{
* Correspondence: Yvonne.Boettcher@medizin.uni-leipzig.de

'IFB AdiposityDiseases, University of Leipzig, Leipzig, Germany

Full list of author information is available at the end of the article
}

triggered by smoking in healthy individuals $[10,12]$ or in cells treated with several toxic chemical compounds such as the antioxidant ethoxiquin or lipid derived aldehydes like HNE (Hydroxynonenal) [13], which are also present in daily food. This suggests protective effects of AKR1B10 against toxic nutrient-specific aldehydes [3,14]. In addition, Akr1b10 expression was shown to be lower in brain of Lewis rats (LEW) that are more sensitive to cocaine or ethanol compared to a control group of rats (Fischer 344 (F344)) [15]. Higueras-Matas et al. (2011) [15] observed a decreased expression of Akr1b10 in nucleus accumbens and the frontal cortex, areas suggested to be involved in eating behavior. Taken together, (i) the important role of AKR1B10 in detoxifying aldehydes and several other deleterious compounds during digestion and (ii) its putative central effects in brain regions potentially involved in eating behavior allowed us to hypothesize that AKR1B10 may play a role in human eating behavior.

There is a good evidence for the role of genetics in human eating behavior [16-19] or in altered eating behavior 
e.g. eating disorders [20]. For instance, risk variants of the fat mass and obesity associated gene (FTO) were not only associated with higher body mass index (BMI) but also with loss of control over eating and selection of food higher in calories and fat [21-23]. Single nucleotide polymorphisms (SNP) in the glutamate decarboxylase gene (GAD2) were significantly associated with increased disinhibition and hunger in women from the Quebec Family Study [24] while a promoter SNP was related to disinhibition and hunger scores in morbidly obese patients from a French cohort [25]. Meyre et al. (2005) further described the promoter variant in GAD2 associated with lower birth weight and subsequently higher BMI Z-Scores in French children [26]. Further, genetic variants within the leptin gene $(L E P)$ and cholecystokinin gene $(C C K)$ were shown to be associated with "snacking behavior" [27] and bigger meal sizes [28]. Dotson and colleagues (2010 \& 2008) [16,29] found that the density of bitter taste receptors is partially determined by genetic factors influencing eating behavior through sensitivity to bitterness. In the present study we investigated SNP markers within the AKR1B10 gene and in the upstream untranslated regulatory region. We evaluated its genetic association with human eating behavior, a variety of metabolic and anthropometric phenotypes, and consumption of consumer goods such as alcohol intake, smoking behavior and coffee consumption. To the best of our knowledge this is the first study investigating $A K R 1 B 10$ variants and their potential relationship with anthropometric and metabolic parameters as well as eating behavior traits.

\section{Methods \\ Subjects \\ Sorbs}

In the present study we used a self-contained population, the Sorbs from Germany, comprising 1046 individuals as described earlier [30] (The entire recruitment was done by Dr. Anke Tönjes and Dr. Jan Halbritter (University of Leipzig, Germany). Sampling was carried out population based. Exclusion criteria were age under 18 years and pregnancy. No specific inclusion criteria in regard to disease states were considered. For subjects recruitment, the study was advertised by flyers, newspapers, radio and by local general practitioners. 618 Sorbs out of 1046 completed the German version of the three factor eating questionnaire (FEV) which determines the three main eating behavior factors - disinhibition, hunger and restraint as described in detail elsewhere $[18,31,32]$. In the here presented study type 2 diabetes (T2D) was the only exclusion criterion because diabetic individuals usually control their food intake according to specific nutrition plans. Therefore, 70 Subjects with T2D have been excluded from the study. Diabetes was diagnosed based on a $75 \mathrm{~g}$ oral glucose tolerance test (OGTT) and the definition of T2D according to ADA criteria [33]. Finally, the study included 548 subjects (346 women and 202 men). No further exclusion criteria were used in this study. All individuals underwent extensive phenotyping $[30,34]$ including a standardized interview for past medical history, family history, measurements of anthropometric parameters (waist, hip, BMI, waist to hip ratio (WHR)), measurements of glucose and insulin metabolism (75 g OGTT, blood glucose and insulin levels at several time points (0,30 and 120 min after meal) [35]) and measurements of lipid metabolism (total cholesterol levels, high and low density lipoprotein cholesterol levels (HDL-C and LDL-C), and triglyceride concentration (TG)) [36]. Insulin was measured with the Auto-DELFIA Insulin assay (PerkinElmer Life and Analytical Sciences, Turku, Finland). Serum glucose was measured by the hexokinase method (Automated analyser Modular, Roche Diagnostics, Mannheim, Germany). Total serum cholesterol and TG concentrations were measured by standard enzymatic methods (CHOD-PAP and GPO-PAP; Roche Diagnostics). Serum HDL and LDL cholesterol concentrations were determined with commercial homogeneous direct measurement methods (Roche Diagnostics). All assays were performed in an automated clinical chemistry analyzer (Hitachi/ Roche Diagnostics) at the Institute of Laboratory Medicine, University Hospital Leipzig. The main characteristics of the participants are summarized in Table 1.

\section{Consumption of consumer goods in sorbs}

Consumption of consumer goods such as coffee and alcohol consumption as well as smoking behavior were assessed by standardized interviews for all subjects (no previously validated questionnaire was used). To estimate coffee and alcohol intake, subjects were asked for average intake but not for a certain time period (alcohol: 1 ) none; 2 ) occasionally at special events; 3 ) 2-3 glasses of wine or bottle of beer per week; 4) 1 glass of wine or bottle of beer per day; 5) 2 glasses of wine or bottles of beer per day; 6) more than 3 glasses of wine or bottles of beer per day; coffee: number of cups per day) [30,37]. We did not account for different contents of alcohol per glass/ bottle or caffeine content per cup. Smoking behavior was obtained by asking for the time period of smoking (currently, former, never) and the number of cigarettes per day. It was not accounted for occasional smokers.

\section{Replication cohort}

For replication purposes we used another independent German cohort as described elsewhere [18] including 350 healthy individuals (152 women and 198 men). Briefly, phenotyping included anthropometric measurements (BMI and weight), age, sex and data for eating behavior, which were assessed using the German version of the three factor eating questionnaire (FEV) [31]. The main characteristics of the subjects are summarized in Table 1 . 
Table 1 Main characteristics of the study populations

\begin{tabular}{|c|c|c|}
\hline & Sorbs & Replication \\
\hline$N$ total & 548 & 350 \\
\hline Age (years) & $48 \pm 16$ & $27 \pm 5$ \\
\hline BMI $\left(k g / m^{2}\right)$ & $26.1 \pm 4.4$ & $27.0 \pm 6.2$ \\
\hline Waist-Hip Ratio & $0.86 \pm 0.1$ & n.a. \\
\hline Waist circumference & $88.39 \pm 13.02$ & n.a. \\
\hline Hip circumference & $102.65 \pm 8.09$ & n.a. \\
\hline Fasting plasma glucose (mmol/l) & $5.24 \pm 0.49$ & n.a. \\
\hline 30 min plasma glucose $(\mathrm{mmol} / \mathrm{l})$ & $8.36 \pm 1.65$ & n.a. \\
\hline 120 min plasme glucose (mmol/l) & $5.41 \pm 1.66$ & n.a. \\
\hline Fasting plasma insulin (pmol/l) & $37.74 \pm 22.71$ & n.a. \\
\hline 30 min plasma insulin (pmol/l) & $290.32 \pm 173.40$ & n.a. \\
\hline 120 plasma glucose (pmol/l) & $173.40 \pm 156.88$ & n.a. \\
\hline Total cholesterol (mmol/l) & $5.34 \pm 1.08$ & n.a. \\
\hline HDL-cholsterol (mmol/l) & $1.69 \pm 0.41$ & n.a. \\
\hline LDL-cholesterol (mmol/l) & $3.37 \pm 0.99$ & n.a. \\
\hline Triglycerides (mmol/l) & $1.23 \pm 0.83$ & n.a. \\
\hline Disinhibition & $4.3 \pm 3.0$ & $6.2 \pm 3.2$ \\
\hline Hunger & $3.9 \pm 2.9$ & $5.5 \pm 3.4$ \\
\hline Restraint & $7.9 \pm 4.8$ & $6.1 \pm 4.4$ \\
\hline Coffee intake (cups per day) & $2 \pm 1$ & n.a \\
\hline Alcohol intake (glasses per week) & $3 \pm 1$ & n.a. \\
\hline Smoking behavior (cigarettes per day) & $10 \pm 8$ & n.a. \\
\hline
\end{tabular}

Data are presented as mean \pm standard deviation (SD) BMI: Body Mass Index; WHR: waist-to-hip ratio. Women/men per population is as follows: Sorbs (346 women, 202 men); Replication (152 women, 198 men) n.a.: not available.

All human studies were approved by the ethics committee of the University of Leipzig and all subjects gave written informed consent.

\section{Genotyping}

Five AKR1B10 SNPs (rs1834150; rs782881; rs3778828; rs4732036; UTR SNP rs10232478) were selected for the analysis. We selected tagging SNPs for our analysis which are in linkage disequilibrium (LD) with several other SNPs within and around the $A K R 1 B 10$ locus to enlarge the probability to find associating variants in the gene without the need to analyze all existing SNPs. The genotype information for rs10232478 was extracted from genome wide Affymetrix SNP data [38] for the Sorbs and individually genotyped in the replication cohort. The remaining variants were taken from the HapMap database $\left(r^{2}>0.8\right.$, minor allele frequency $(\mathrm{MAF})<0.05)$ and genotyped using the KASPar SNP genotyping system (KBiosiences Ltd., Hoddesdon, United Kingdom) according to manufacturer's protocol (TD PCR (touch down polymerase chain reaction) with following conditions: $94^{\circ} \mathrm{C}$ for $15 \mathrm{sec}, 94^{\circ} \mathrm{C}$ for $20 \mathrm{sec}, 61^{\circ} \mathrm{C}$ for $60 \mathrm{sec}$, for 10 cycles, drop down $0.6^{\circ} \mathrm{C}$ per cycle, $94^{\circ} \mathrm{C}$ for $10 \mathrm{sec}, 55^{\circ} \mathrm{C}$ for $60 \mathrm{sec}$, for $26 \mathrm{cycles}$ ).
Genomic deoxyribonucleic acid (DNA) for genotyping was extracted from blood with QIAmp DNA Blood Midi Kit (Qiagen, Hilden, Germany) according to the manufacturer's protocol. Fluorescence was detected using ABI PRISM 7500 Sequence Detecting System. To guarantee genotyping reproducibility, a random $\sim 5 \%$ of the samples were re-genotyped in all SNPs; all genotypes matched initial designated genotypes. The average genotyping success rate for all analyzed SNPs was $96.38 \%$.

\section{Statistical analysis}

Statistical analyses were performed using the SPSS statistics software version 20.0 (SPSS, Inc.; Chicago, IL). Nonnormally distributed parameters were $\log _{10}$-transformed to approximate normal distribution prior to analysis. All variants were in Hardy-Weinberg-Equilibrium. Genetic association with eating behavior traits, anthropometric and metabolic phenotypes were calculated using linear regression models adjusted for age, gender and lnBMI (except for BMI). Cronbach's alpha coefficients were calculated to measure the internal reliability of the three eating behavior subscales. Coffee consumption was defined as cups per day, smoking behavior as number of cigarettes per day and alcohol intake as number of glasses per week. We did not account for different contents of alcohol per glass/bottle or caffeine content per cup. $P$-values for smoking, alcohol intake and coffee consumption were corrected for age and gender. Additive, dominant and recessive models of inheritance were tested. To correct for multiple testing we lowered the significance threshold to $\left(0.05 /(315\right.$ tests $\left.)=1.6 \times 10^{-4}\right)$. All $P$-values $>1.6 \times 10^{-4}$ but $\leq 0.05$ were considered to be of nominal statistical significance. All $P$-values are provided uncorrected for multiple testing. Meta-analyses were performed using METAL [39]. All analyses were standardized to the minor allele (plus strand).

\section{Results}

\section{Association analysis in sorbs}

We observed nominally significant associations with disinhibition at the variant rs10232478 located in the $5^{\prime}$ untranslated region and the intronic SNPs rs1834150 and rs782881 (Table 2). For each of the variants the minor alleles were related to increased disinhibition scores. A trend for nominal association with restraint scores was shown at rs3778828. No relationships were obtained for any of the variants with hunger (Table 2).

Furthermore, we observed decreased waist circumference in homozygous minor allele carriers when applying recessive inheritance models, respectively (rs782881 $P=0.001$; rs1834150 $P=0.004$; Table 2 ). A slightly increased coffee consumption was detected in homozygous or heterozygous minor allele carriers of rs1834150 $(P=0.043$; Table 2$)$. Further, while carriers of the minor 
Table 2 Association analysis of AKR1B10 variants in the Sorbs population

\begin{tabular}{|c|c|c|c|c|c|c|c|c|c|c|c|c|c|c|c|}
\hline \multicolumn{16}{|c|}{ AKR1B10 genotype } \\
\hline \multirow[b]{2}{*}{ Genotype $(N)$} & \multicolumn{3}{|l|}{ rs 10232478} & \multicolumn{3}{|l|}{ rs782881 } & \multicolumn{3}{|l|}{ rs1834150 } & \multicolumn{3}{|l|}{ rs3778828 } & \multicolumn{3}{|l|}{ rs4732036 } \\
\hline & CC (76) & CT (230) & TT (195) & CC (114) & AC (263) & AA (150) & $\pi(115)$ & TA (257) & AA (171) & AA (37) & GA (201) & GG (293) & CC (49) & TC (210) & $\Pi(280)$ \\
\hline \multicolumn{16}{|c|}{ Association analysis with anthropometric traits } \\
\hline BMI $\left(\mathrm{kg} / \mathrm{m}^{2}\right)$ & $25.96 \pm 3.83$ & $26.42 \pm 4.94$ & $25.99 \pm 4.09$ & $26.5 \pm 4.9$ & $25.9 \pm 4.4$ & $26.2 \pm 4.1$ & $26.2 \pm 4.1$ & $26 \pm 4.5$ & $26.1 \pm 4.1$ & $26.4 \pm 4$ & $25.9 \pm 4$ & $26.2 \pm 4.7$ & $26.0 \pm 4.9$ & $25.9 \pm 4.2$ & $26.1 \pm 4.4$ \\
\hline p-value & n.s. & & & n.s. & & & n.s. & & & n.s. & & & n.s. & & \\
\hline Waist (cm) & $87.17 \pm 11.70$ & $88.92 \pm 13.6$ & $88.91 \pm 13.37$ & $87.80 \pm 13.1$ & $88.22 \pm 12.8$ & $89.18 \pm 13.3$ & $87.20 \pm 11.8$ & $88.64 \pm 13.2$ & $88.76 \pm 13.2$ & $87.78 \pm 12.6$ & $88.60 \pm 12.8$ & $88.20 \pm 13.2$ & $88.12 \pm 14.9$ & $88.46 \pm 12.9$ & $88.33 \pm 12.8$ \\
\hline$p$-value & n.s. & & & "0.001 & & & "0.004 & & & n.s. & & & n.s. & & \\
\hline Hip (cm) & $101.6 \pm 7.3$ & $103.6 \pm 9.2$ & $102.4 \pm 7.4$ & $102.61 \pm 9.1$ & $106.22 \pm 55.8$ & $102.77 \pm 7.4$ & $102.37 \pm 7.8$ & $106.39 \pm 56.4$ & $102.39 \pm 7.4$ & $104.14 \pm 7.5$ & $102.17 \pm 7$ & $105.99 \pm 53.0$ & $102.35 \pm 9.4$ & $106.67 \pm 62.3$ & $102.82 \pm 7.9$ \\
\hline$p$-value & n.s. & & & n.s. & & & n.s. & & & n.s. & & & n.s. & & \\
\hline WHR & $0.86 \pm 0.09$ & $0.86 \pm 0.09$ & $0.87 \pm 0.1$ & $0.85 \pm 0.1$ & $0.85 \pm 0.1$ & $0.87 \pm 0.1$ & $0.85 \pm 0.1$ & $0.86 \pm 0.1$ & $0.86 \pm 0.1$ & $0.84 \pm 0.1$ & $0.86 \pm 0.1$ & $0.85 \pm 0.1$ & $0.86 \pm 0.1$ & $0.86 \pm 0.1$ & $0.86 \pm 0.1$ \\
\hline p-value & n.s. & & & n.s. & & & n.s. & & & n.s. & & & n.s. & & \\
\hline \multicolumn{16}{|c|}{ Association analysis with eating behavior factors } \\
\hline Disinhibition & $4.95 \pm 2.88$ & $4.53 \pm 3.16$ & $3.81 \pm 2.7$ & $4.9 \pm 3.11$ & $4.3 \pm 3.02$ & $4.04 \pm 2.92$ & $4.93 \pm 3.16$ & $4.33 \pm 2.95$ & $3.94 \pm 2.89$ & $4.65 \pm 3.04$ & $4.25 \pm 2.99$ & $4.41 \pm 3.03$ & $4.29 \pm 2.92$ & $4.29 \pm 3.12$ & $4.43 \pm 2.94$ \\
\hline$p$-value & ${ }^{\circ} 0.003$ & & & ${ }^{\circ} 0.0320 .03$ & & & ${ }^{\circ} 0.006 \# 0.02$ & $27 " 0.022$ & & n.s. & & & n.s. & & \\
\hline Hunger & $4.09 \pm 2.94$ & $4.04 \pm 2.84$ & $3.66 \pm 2.66$ & $4.26 \pm 2.86$ & $3.83 \pm 2.86$ & $3.92 \pm 3.10$ & $4.17 \pm 2.88$ & $3.85 \pm 2.85$ & $3.95 \pm 3.04$ & $3.99 \pm 3.16$ & $3.99 \pm 2.81$ & $3.93 \pm 2.98$ & $3.84 \pm 2.8$ & $4.07 \pm 3.05$ & $3.88 \pm 2.86$ \\
\hline p-value & n.s. & & & n.s. & & & n.s. & & & n.s. & & & n.s. & & \\
\hline Restraint & $8.79 \pm 5.32$ & $7.86 \pm 4.73$ & $7.62 \pm 4.92$ & $8.52 \pm 5.13$ & $7.71 \pm 4.67$ & $7.65 \pm 5.05$ & $8.52 \pm 5.09$ & $7.82 \pm 4.77$ & $7.6 \pm 4.9$ & $7.42 \pm 5.03$ & $7.53 \pm 4.88$ & $8.31 \pm 4.83$ & $7.8 \pm 4.37$ & $7.57 \pm 4.88$ & $8.24 \pm 4.96$ \\
\hline p-value & n.s. & & & n.s. & & & n.s. & & & ${ }^{\circ} 0.048$ & & & n.s. & & \\
\hline \multicolumn{16}{|c|}{ Association analysis with consumer goods } \\
\hline Smoking & $10.04 \pm 8.79$ & $10.07 \pm 8.13$ & $9.45 \pm 8.09$ & $9.91 \pm 8.9$ & $10.34 \pm 8.18$ & $8.4 \pm 7.4$ & $10.6 \pm 9.31$ & $9.58 \pm 7.95$ & $10.02 \pm 8.48$ & $4.67 \pm 3.33$ & $10.32 \pm 8.98$ & $9.89 \pm 8.23$ & $11.94 \pm 8.44$ & $9.39 \pm 8.35$ & $9.89 \pm 8.52$ \\
\hline$p$-value & n.s. & & & $\# 0.044$ & & & n.s. & & & n.s. & & & n.s. & & \\
\hline Alcohol & $2.83 \pm 1.2$ & $2.78 \pm 1.17$ & $2.82 \pm 1.2$ & $2.88 \pm 1.18$ & $2.76 \pm 1.11$ & $2.79 \pm 1.28$ & $2.84 \pm 1.23$ & $2.77 \pm 1.12$ & $2.79 \pm 1.23$ & $2.84 \pm 1.30$ & $2.8 \pm 1.22$ & $2.77 \pm 1.14$ & $2.9 \pm 1.19$ & $2.82 \pm 1.19$ & $2.75 \pm 1.17$ \\
\hline p-value & n.s. & & & n.s. & & & n.s. & & & n.s. & & & n.s. & & \\
\hline Coffee & $1.79 \pm 0.72$ & $1.79 \pm 0.76$ & $1.74 \pm 0.75$ & $1.81 \pm 0.76$ & $1.89 \pm 0.82$ & $1.75 \pm 0.75$ & $1.81 \pm 0.73$ & $1.89 \pm 0.82$ & $1.71 \pm 0.75$ & $1.62 \pm 0.68$ & $1.84 \pm 0.82$ & $1.81 \pm 0.76$ & $1.71 \pm 0.61$ & $1.84 \pm 0.79$ & $1.80 \pm 0.80$ \\
\hline$p$-value & n.s. & & & n.s. & & & $\# 0.043$ & & & n.s. & & & n.s. & & \\
\hline
\end{tabular}

Data are presented as mean \pm SD. Type 2 diabetics were excluded. $P$-values were calculated using additive, dominant $(\mathrm{mm}+\mathrm{mM}$ vs. $\mathrm{MM})$ and recessive $(\mathrm{mm}$ vs $\mathrm{mM}+\mathrm{MM}) \mathrm{model}$ of inheritance using linear regression adjusted for age, gender and InBMI (except for BMI). Analyses for smoking, alcohol and coffee consumption were adjusted for age and gender; ${ }^{\circ}=$ additive model of inheritance; \# $=$ dominant model of inheritance; "=recessive model of inheritance; $n$.s. non-significant; significant $P$-values are highlighted in bold; $N=$ number of individuals; BMI = Body Mass Index; WHR = Waist-Hip-Ratio. 
C-allele of rs782881 showed higher cigarette consume per day (dominant model, $P=0.044$; Table 2), we observed no further relationship to the time period of smoking.

Additional analyses were performed to test the hypothesis that variants within $A K R 1 B 10$ are related to glucose, insulin and lipid metabolism. Beside a few nominal associations of rs10232478, rs1834150, rs3778828 and rs4732036 with fasting plasma insulin values, 120 min plasma insulin values, triglycerides levels and 120 min plasma glucose levels (respectively), we did not detect evidence for any significant associations (Additional file 1: Table S1). None of the here reported $P$-values withstand correction for multiple testing (Bonferroni).

\section{Association analysis in the replication cohort}

In order to verify our results we performed linear regression analyses for rs10232478; rs782881 and rs1834150 in an independent German cohort $(N=350)$. Despite failing to reach statistical significance our replication analyses revealed similar trends for higher disinhibition scores in homozygous or heterozygous minor allele carriers (Table 3 ). We observed a similar effect direction as compared to the Sorbs at rs1834150 and a meta-analysis for rs1834150 resulted in a combined $P=0.0096$ (Table 4).

Interestingly, when analyzing a potential correlation of the AKR1B10 SNPs with restraint scores we observed a nominally significant relationship with increased restraint at the same intragenic variants (rs1834150; rs782881) and the 5' UTR variant rs10232478 (all $P \leq 0.05$; Table 3). In a meta-analysis including the Sorbs and the replication cohort we confirmed a nominally significant association of rs1834150 ( $P=0.00728$, Table 4$)$ with restraint. Moreover, we observed a nominal association of rs10232478 in the $5^{\prime}$ untranslated region with the eating behavior factor hunger $(P \leq 0.05$; Table 3$)$.

\section{Discussion}

The human AKR1B10 was shown to be associated with several types of cancers including lung cancer [10] and liver cancer [9]. AKR1B10 is highly expressed in the gastrointestinal tract [1] and its expression is triggered via toxic aldehydes like acrolein and HNE present in many daily nutrients $[3,13]$ or through cigarette smoke $[10,12]$. The reductase AKR1B10 is highly reactive towards this components and may lead to their detoxification during digestion [14]. Interestingly, Higueras-Matas and colleagues found an inhibition of $a k r 1 b 10$ gene expression in rats more sensitive to drugs like cocaine and ethanol in brain regions known to regulate eating behavior [15]. While a potential role of AKR1B10 in eating behavior seems plausible in the light of these studies, its role in BMI, waist and hip circumference or the influence on metabolic parameters was never analyzed so far. In the present study we therefore hypothesized AKR1B10 to play a role in human eating behavior and tested for a relationship to metabolic and anthropometric variables. Using linear regression models we observed a relationship of genetic variants within $A K R 1 B 10$ and disinhibition scores in the Sorbs along with similar directions in the replication cohort. Disinhibition is a measure of the loss of cognitive control during eating due to situational factors [40]. This may result in overeating and a higher caloric intake potentially contributing to an increase in body weight [41]. Most of the studies suggest a positive association between high disinhibition levels, BMI and weight gain $[42,43]$. In contrast, we did not identify any relationship to BMI but observed indications for lower waist

Table 3 Association analysis of $A K R 1 B 10$ variants in the replication cohort

\begin{tabular}{|c|c|c|c|c|c|c|c|c|c|}
\hline \multicolumn{10}{|c|}{ AKR1B10 genotype } \\
\hline \multirow[b]{2}{*}{ Genotype $(N)$} & \multicolumn{3}{|l|}{ rs10232478 } & \multicolumn{3}{|l|}{ rs782881 } & \multicolumn{3}{|l|}{ rs1834150 } \\
\hline & CC (50) & CT (131) & TT (99) & CC (65) & AC (146) & AA (81) & $\mathrm{TT}(56)$ & TA (136) & AA (82) \\
\hline age & $27.14 \pm 5.46$ & $26.40 \pm 4.87$ & $26.53 \pm 4.71$ & $26.80 \pm 5.54$ & $26.64 \pm 4.97$ & $26.91 \pm 4.84$ & $26.70 \pm 4.82$ & $26.43 \pm 5.03$ & $27.50 \pm 5.01$ \\
\hline p-value & n.s. & & & n.s. & & & n.s. & & \\
\hline BMl & $27.6 \pm 6.53$ & $26.92 \pm 6.16$ & $27.21 \pm 6.49$ & $27.64 \pm 6.67$ & $26.90 \pm 5.93$ & $27.36 \pm 6.59$ & $26.98 \pm 5.94$ & $27.34 \pm 6.20$ & $27.11 \pm 6.59$ \\
\hline p-value & n.s. & & & n.s. & & & n.s. & & \\
\hline \multicolumn{10}{|c|}{ association analysis with eating behavior factors } \\
\hline Disinhibition & $6.64 \pm 3.34$ & $6.05 \pm 3.12$ & $6.33 \pm 3.02$ & $6.15 \pm 3.11$ & $6.33 \pm 3.22$ & $6.01 \pm 3.29$ & $6.29 \pm 3.06$ & $6.56 \pm 3.31$ & $6.01 \pm 3.21$ \\
\hline$p$-value & n.s. & & & n.s. & & & n.s. & & \\
\hline Hunger & $4.63 \pm 2.87$ & $5.57 \pm 3.48$ & $5.88 \pm 3.26$ & $4.80 \pm 3.3$ & $5.72 \pm 3.51$ & $5.76 \pm 3.42$ & $4.72 \pm 3.18$ & $5.99 \pm 3.57$ & $5.54 \pm 3.37$ \\
\hline p-value & \multicolumn{3}{|c|}{${ }^{\circ} 0.038$ "0.036 } & \multicolumn{3}{|l|}{ n.s. } & \multicolumn{3}{|l|}{ n.s. } \\
\hline Restraint & $7.85 \pm 5.23$ & $6.30 \pm 4.41$ & $5.55 \pm 4.23$ & $7.27 \pm 4.91$ & $6.16 \pm 4.22$ & $5.42 \pm 4.29$ & $7.35 \pm 5.14$ & $5.99 \pm 4.13$ & $5.25 \pm 4.06$ \\
\hline$p$-value & \multicolumn{3}{|c|}{${ }^{\circ} 0.010$ "0.015 } & \multicolumn{3}{|l|}{${ }^{\circ} 0.027$} & \multicolumn{3}{|c|}{${ }^{\circ} 0.007$ \#0.049 0.015 } \\
\hline
\end{tabular}

Data are presented as mean \pm SD. Type 2 diabetics were excluded. $P$-values were calculated using additive, dominant $(\mathrm{mm}+\mathrm{mM}$ vs. $\mathrm{MM})$ and recessive $(\mathrm{mm}$ vs $\mathrm{mM}+\mathrm{MM})$ model of inheritance using linear regression adjusted for age, sex and InBMI (except for BMI); ${ }^{\circ}=$ additive model of inheritance; $\#=$ dominant model of inheritance;

“=recessive model of inheritance; n.s. non-significant; significant $P$-values are highlighted in bold; $N=$ number of individuals; $\mathrm{BMI}=\mathrm{Body}$ Mass Index. 
Table 4 Meta-analysis for association with eating behavior factors

\begin{tabular}{|c|c|c|c|c|c|c|c|c|c|c|}
\hline \multirow[b]{2}{*}{ SNP } & \multirow[b]{2}{*}{ Minor allele } & \multicolumn{3}{|l|}{ Sorbs } & \multicolumn{3}{|c|}{ German cohort } & \multicolumn{3}{|c|}{ Combined } \\
\hline & & $P$-value & $\beta$ & S.E. & $P$-value & $\beta$ & S.E. & P-value & Z-score & Direction \\
\hline & & \multicolumn{9}{|c|}{ Disinhibition } \\
\hline rs10232478 & C & 0.003 & +0.071 & 0.024 & 0.948 & -0.016 & 0.248 & 0.0194 & +2.336 & +- \\
\hline rs782881 & C & 0.032 & +0.357 & 0.167 & 0.977 & -0.007 & 0.243 & 0.0888 & +1.702 & +- \\
\hline \multirow[t]{2}{*}{ rs1834150 } & $\mathrm{T}$ & 0.006 & +0.439 & 0.160 & 0.544 & +0.153 & 0.252 & 0.0096 & +2.591 & ++ \\
\hline & & \multicolumn{9}{|c|}{ Restraint } \\
\hline rs10232478 & C & 0.334 & +0.040 & 0.042 & 0.010 & +0.993 & 0.385 & 0.0211 & +2.306 & ++ \\
\hline rs782881 & C & 0.185 & +0.381 & 0.287 & 0.027 & +0.816 & 0.367 & 0.0174 & +2.378 & ++ \\
\hline \multirow[t]{2}{*}{ rs1834150 } & $\mathrm{T}$ & 0.166 & +0.382 & 0.276 & 0.007 & +1.006 & 0.372 & 0.0072 & +2.684 & ++ \\
\hline & & \multicolumn{9}{|l|}{ Hunger } \\
\hline rs10232478 & C & 0.209 & +0.030 & 0.024 & 0.038 & -0.589 & 0.282 & 0.818 & -0.230 & +- \\
\hline rs782881 & C & 0.542 & +0.106 & 0.174 & 0.092 & -0.487 & 0.288 & 0.6081 & -0.513 & +- \\
\hline rs1834150 & $\mathrm{T}$ & 0.785 & +0.046 & 0.168 & 0.237 & -0.357 & 0.301 & 0.6459 & -0.459 & +- \\
\hline
\end{tabular}

$P$-values were calculated based on effect sizes from linear regression model using additive inheritance model. Significant $P$-values withstanding correction for multiple testing are presented in bold. All data are adjusted for age, gender and InBMI. All analyses were standardized to the minor allele.

circumference at three SNPs which are related to disinhibition. This finding may be explained by the fact that restraint scores at rs1834150, rs782881 and rs10232478 are increased as well. Restraint eaters tend to rigorously control their body weight by permanently controlling their eating habits. These individuals often count calories in order to regulate the amount of food intake [40]. Since both, restraint and disinhibition, are not completely independent, the observed increased restraint scores could probably serve as a counteracting behavior against the disinhibition effect $[40,44]$. One may argue that high restraint scores were also found to be associated with a higher BMI [45]. However, it needs to be mentioned that restraint is not a homogeneous measure but can be divided in two subscales - rigid control ('all or nothing') and flexible control ('more or less') $[45,46]$. While rigid control is associated with higher BMI and eating disorders, flexible restraint is related to decreased BMI and weight loss $[43,46]$. Furthermore, it was demonstrated that individuals presenting rigid control show increased restraint scores and simultaneously high disinhibition scores which results in increased weight or BMI. In contrast, flexible cognitive control was associated with decreased BMI and weight $[41,43,47]$. We did not have the opportunity to analyze restraint subscales, but nevertheless our data indicate a counteracting mechanism between disinhibition and restraint which results in a decrease of waist circumference, probably due to a highly flexible cognitive control. Moreover, we found rs1834150 and rs782881 related to intake of consumer goods in the Sorbs. In particular, we show a nominal association at rs1834150 and higher coffee consumption per day and found rs782881 correlated with an increased amount of cigarettes per day. These findings, together with our results from the eating behavior questionnaire FEV, suggest a potential role of $A K R 1 B 10$ in human eating behavior. However, it needs to be acknowledged that none of the here observed relationships withstand a Bonferroni correction for multiple testing, thus underscoring the possibility of false positive findings. Therefore, although our data may indicate a role of $A K R 1 B 10$ in eating behavior, intake of consumer goods and waist circumference, they need to be interpreted with caution.

Further, we tested for a potential relationship of the $A K R 1 B 10$ genetic variants to various variables of the glucose and insulin metabolism as well as lipid profiles. Beside several, rather occasional, non-significant correlations we did not identify any consistent evidence for significant associations with the tested SNPs.

Despite some interesting results, our study is limited at several aspects. (i) Although we present a second independent replication cohort the sample size of our study is rather small which may lead to false positive and false negative results. (ii) There is a large discrepancy in the male/female ratio and in age between the study cohorts which may have, in terms of different eating behavior patterns between men and women, influenced our results as demonstrated by others [48-50]. However, we used gender as covariate in our analysis whereby the influence of discrepancy in the male/female ratio on the results was taken into account. (iii) Due to the lack of data for metabolic variables and consumer goods in the replication cohort we cannot conduct meta-analysis for both sample sets. (iv) While the German version of the three factor eating questionaire (FEV) is validated as reported by Pudel V \& Westenhoefer J [31] with internal reliability of the three scales (between $\mathrm{r}=0.75$ and 0.87 ) and similar data on internal reliability observed in the Sorbs (Cronbach's alpha coefficients: disinhibition $=0.744$; restraint $=0.856$; 
hunger $=0.745$ ), questions related to consumer goods were not validated. Therefore, our data on consumer goods need to be interpreted with caution.

In conclusion, our data in regard to eating behavior factors support our hypothesis that AKR1B10 may be a potential contributor to human eating behavior. However, our data need to be cautiously interpreted and larger studies are definitely warranted to replicate and verify the observed effects.

\section{Conclusions}

To the best of our knowledge this is the first evidence for an association of AKR1B10 with eating behavior. The established role of AKR1B10 in cancers via influencing important signaling pathways like the retinoid acid metabolism $[6,8]$ together with the here presented potential role in human eating behavior may give rise to further scenarios through which AKR1B10 may function in other pathways.

\section{Additional file}

Additional file 1: Table S1. Association analysis of AKR1B10 variants in the Sorbs population.

\section{Abbreviations}

AKR1B10: Human Aldoketoreductase 1 B10 gene; mRNA: Messenger ribonucleic acid; 5' UTR: 5' Untranslated region; NADP(H): Nicotinamide adenine dinucleotide phosphate; HNE: Hydroxynonenal; LEW: Lewis rats; F344: Fischer344 rats; BMI: Body mass index; FTO: Fat mass and obesity associated gene; SNP: Single nucleotide polymorphism; GAD2: Glutamate decarboxylase gene; LEP: Leptin gene; CCK: Cholecystokinin gene; FEV: German version of the three factor eating questionnaire; T2D: Type 2 diabetes; OGTT: Oral glucose tolerance test; HDL-C: High density lipoprotein cholesterol; LDL-C: Low density lipoprotein cholesterol; TG: Triglycerides; LD: Linkage disequilibrium; MAF: Minor allele frequency; TD PCR: Touch down polymerase chain reaction; DNA: Deoxyribonucleic acid; N: Number; SD: Standard deviation; n.a.: Not available.
\end{abstract}

\section{Competing interests}

The authors declare that they have no competing interests.

\section{Authors' contributions}

$K R$, YB conceived and designed the experiments; KR analyzed the data; MF, $\mathrm{AH}, \mathrm{MK}$, contributed reagents/materials/analysis tools; $\mathrm{KR}$ and $\mathrm{YB}$ wrote the first draft of the manuscript; MF, AH, MK, AV, MS, AT, PK, YB edited the manuscript and contributed to the final manuscript version. All authors read and approved the final manuscript.

\footnotetext{
Acknowledgement

We thank all those who participated in the studies. We are grateful to Marie-Therese Gast for excellent work. This project was supported by grants from the DFG (BO/3147-4 to Y.B.), the German Diabetes Association (to Y.B., A.T., P.K.) and from the DDS Foundation to Y.B. K.R. is funded by a research grant from the Boehringer Ingelheim Foundation. The work was further supported by the IFB AdiposityDiseases (ADI-K50D, ADI-K7-38, ADI-K7-39, ADI-K7-45 to Y.B. and ADI-K60E, ADI-K7-57 to P.K.) and K7-37 and K403 (both to A.T.). IFB AdiposityDiseases is supported by the Federal Ministry of Education and Research (BMBF), Germany, FKZ: 01EO1001. M.K. is funded by an EFSD/Lilly research fellowship (to Y.B.). A.H., M.S., A.T. A.V. and P.K. are supported by a Collaborative Research Center granted by the DFG (CRC 1052: "Obesity Mechanisms"; A05 to A.H.; A01 and C01 to M.S.; C01 to A.T.; A01 to A.V. and to B03 P.K.).
}

\section{Author details}

${ }^{1}$ IFB AdiposityDiseases, University of Leipzig, Leipzig, Germany. ${ }^{2}$ Max-Planck-Institute for Human Cognitive and Brain Sciences, Department of Neurology, Leipzig, Germany. ${ }^{3}$ Department of Medicine, University of Leipzig, Leipzig, Germany. ${ }^{4}$ Clinic of Cognitive Neurology, University of Leipzig, Leipzig, Germany.

Received: 13 March 2014 Accepted: 11 March 2015

Published online: 25 March 2015

\section{References}

1. Cao D. Identification and characterization of a novel human aldose reductase-like gene. J Biol Chem. 1998;273(19):11429-35.

2. Petrash JM. All in the family: aldose reductase and closely related aldo-keto reductases. Cell Mol Life Sci. 2004;61(7-8):737-49.

3. Shen $Y$, Zhong L, Johnson S, Cao D. Human aldo-keto reductases $1 B 1$ and 1B10: a comparative study on their enzyme activity toward electrophilic carbonyl compounds. Chem Biol Interact. 2011;191(1-3):192-8.

4. Wang C, Yan R, Luo D, Watabe K, Liao D, Cao D. Aldo-keto reductase family 1 member B10 promotes cell survival by regulating lipid synthesis and eliminating carbonyls. J Biol Chem. 2009:284(39):26742-8.

5. Ma J, Yan R, Zu X, Cheng J, Rao K, Liao D, et al. Aldo-keto reductase family 1 B10 affects fatty acid synthesis by regulating the stability of Acetyl-CoA Carboxylase- in breast cancer cells. J Biol Chem. 2007;283(6):3418-23.

6. Ruiz FX, Gallego O, Ardèvol A, Moro A, Domínguez M, Alvarez S, et al. Aldo-keto reductases from the AKR1B subfamily: retinoid specificity and control of cellular retinoic acid levels. Chem Biol Interact. 2009;178(1-3):171-7.

7. Ruiz FX, Porté S, Parés X, Farrés J. Biological role of Aldo-Keto reductases in retinoic acid biosynthesis and signaling. Front Pharmacol. 2012; 3:10.3389/fphar.2012.00058.

8. Matsunaga T, Wada Y, Endo S, Soda M, El-Kabbani O, Hara A. Aldo-Keto reductase $1 \mathrm{~B} 10$ and its role in proliferation capacity of drug-resistant cancers. Front Pharmacol. 2012;3:10.3389/fphar.2012.00005.

9. Schmitz KJ, Sotiropoulos GC, Baba HA, Schmid KW, Müller D, Paul A, et al. AKR1B10 expression is associated with less aggressive hepatocellular carcinoma: a clinicopathological study of 168 cases. Liver Int. 2011;31(6):810-6.

10. Kang M, Lee E, Yoon S, Jo J, Lee J, Kim H, et al. AKR1B10 is associated with smoking and smoking-related Non-small-cell lung cancer. J Int Med Res. 2011;39(1):78-85.

11. Ma J, Luo D, Huang C, Shen Y, Bu Y, Markwell S, et al. AKR1B10 overexpression in breast cancer: association with tumor size, lymph node metastasis and patient survival and its potential as a novel serum marker. Int J Cancer. 2012;131(6):E862-71.

12. Wang R. Smoking-induced upregulation of AKR1B10 expression in the airway epithelium of healthy individuals. CHEST. 2010;138(6):1402.

13. Nishinaka T, Miura T, Okumura M, Nakao F, Nakamura H, Terada T. Regulation of aldo-keto reductase AKR1B10 gene expression: involvement of transcription factor Nrf2. Chem Biol Interact. 2011;191(1-3):185-91.

14. Pastel E, Pointud J, Volat F, Martinez A, Lefrançois-Martinez A. Aldo-Keto Reductases 1B in endocrinology and metabolism. Front Pharmacol. 2012:3:10.3389/fphar.2012.00148.

15. Higuera-Matas A, Montoya LG, Coria MS, Miguens M, Garcia-Lecumberri C, Ambrosio E. Differential gene expression in the nucleus accumbens and frontal cortex of Lewis and Fischer 344 rats relevant to drug addiction. CN. 2011;9(1):143-50.

16. Dotson CD, Shaw HL, Mitchell BD, Munger SD, Steinle NI. Variation in the gene TAS2R38 is associated with the eating behavior disinhibition in Old Order Amish women. Appetite. 2010;54(1):93-9.

17. Grimm ER, Steinle NI. Genetics of eating behavior: established and emerging concepts. Nutr Rev. 2011;69(1):52-60.

18. Gast M, Tönjes A, Keller M, Horstmann A, Steinle N, Scholz M, et al. The role of rs2237781 within GRM8 in eating behavior. Brain Behav. 2013;3(5):495-502.

19. Tanaka T, Ngwa JS, van Rooij FJ, Zillikens MC, Wojczynski MK, Frazier-Wood AC, et al. Genome-wide meta-analysis of observational studies shows common genetic variants associated with macronutrient intake. Am J Clin Nutrs. 2013;97(6):1395-402.

20. Boraska V, Davis OS, Cherkas LF, Helder SG, Harris J, Krug I, et al. Genome-wide association analysis of eating disorder-related symptoms, behaviors, and personality traits. Am J Med Genet. 2012;159B(7):803-11. 
21. Tanofsky-Kraff M, Han JC, Anandalingam K, Shomaker LB, Columbo KM, Wolkoff LE, et al. The FTO gene rs9939609 obesity-risk allele and loss of control over eating. Am J Clin Nutr. 2009;90(6):1483-8.

22. Park SL, Cheng I, Pendergrass SA, Kucharska-Newton AM, Lim U, Ambite JL, et al. Association of the FTO obesity risk variant rs8050136 with percentage of energy intake from fat in multiple racial/ethnic populations: the PAGE study. Am J Epidemiol. 2013;178(5):780-90.

23. Dougkas A, Yaqoob P, Givens DI, Reynolds CK, Minihane AM. The impact of obesity-related SNP on appetite and energy intake. Br J Nutr. 2013;110(06):1151-6.

24. Choquette AC, Lemieux S, Tremblay A, Drapeau V, Bouchard C, Vohl M, et al. GAD2 gene sequence variations are associated with eating behaviors and weight gain in women from the Quebec family study. Physiol Behav. 2009;98(4):505-10.

25. Boutin $P$, Dina $C$, Vasseur F, Dubois $S$, Corset $L$, Séron $K$, et al. GAD2 on chromosome 10p12 is a candidate gene for human obesity. Plos Biol. 2003;1(3):e8.

26. Meyre D, Boutin P, Tounian A, Deweirder M, Aout $M$, Jouret B, et al. Is glutamate decarboxylase 2 (GAD2) a genetic link between low birth weight and subsequent development of obesity in children? J Clin Endocrinol Metab. 2005:90(4):2384-90.

27. Appelhans BM. Circulating leptin moderates the effect of stress on snack intake independent of body mass. Eat Behav. 2010;11(3):152-5.

28. De KM, Van der Schouw YT, Hendriks J, Ophoff RA, Van Gils CH, Stolk RP, et al. Common genetic variations in CCK, leptin, and leptin receptor genes are associated with specific human eating patterns. Diabetes. 2007:56((1):276-80

29. Dotson CD, Zhang L, Xu H, Shin Y, Vigues S, Ott SH, et al. Bitter taste receptors influence glucose homeostasis. PLoS One. 2008;3(12):e3974.

30. Tönjes A, Zeggini E, Kovacs P, Böttcher Y, Schleinitz D, Dietrich K, et al. Association of FTO variants with BMI and fat mass in the self-contained population of Sorbs in Germany. Eur J Hum Genet. 2009;18(1):104-10.

31. Pudel V, Westenhoefer J. Fragebogen zum Eßverhalten (FEV). 1989

32. Breitfeld J, Tönjes A, Gast M, Schleinitz D, Blüher M, Stumvoll M, et al. Role of vaspin in human eating behaviour. PLoS One. 2013;8(1):e54140.

33. American Diabetes Association. Diagnosis and classification of diabetes mellitus. Diabetes Care. 2009;33(Supplement_1):S62-9.

34. Veeramah KR, Tönjes A, Kovacs $P$, Gross A, Wegmann D, Geary $P$, et al. Genetic variation in the Sorbs of eastern Germany in the context of broader European genetic diversity. Eur J Hum Genet. 2011;19(9):995-1001.

35. Bottcher $Y$, Unbehauen $H$, Kloting N, Ruschke K, Korner A, Schleinitz D, et al. Adipose tissue expression and genetic variants of the bone morphogenetic protein receptor 1A gene (BMPR1A) are associated with human obesity. Diabetes. 2009;58(9):2119-28

36. Keller M, Schleinitz D, Forster J, Tonjes A, Bottcher Y, Fischer-Rosinsky A, et al. THOC5: a novel gene involved in HDL-cholesterol metabolism. J Lipid Res. 2013;54(11):3170-6.

37. Sulem P, Gudbjartsson DF, Geller F, Prokopenko I, Feenstra B, Aben KKH, et al. Sequence variants at CYP1A1-CYP1A2 and AHR associate with coffee consumption. Hum Mol Genet. 2011;20(10):2071-7.

38. Tonjes A, Koriath $\mathrm{M}$, Schleinitz D, Dietrich $\mathrm{K}$, Bottcher $\mathrm{Y}$, Rayner NW, et al. Genetic variation in GPR133 is associated with height: genome wide association study in the self-contained population of Sorbs. Hum Mol Genet. 2009;18(23):4662-8.

39. Willer CJ, Li Y, Abecasis GR. METAL: fast and efficient meta-analysis of genomewide association scans. Bioinformatics. 2010;26(17):2190-1.

40. Westenhoefer J, Broeckmann P, Münch A, Pudel V. Cognitive control of eating behaviour and the disinhibition effect. Appetite. 1994;23(1):27-41.

41. Gallant AR, Tremblay A, Pérusse L, Bouchard C, Després J, Drapeau V. The Three-Factor Eating Questionnaire and BMI in adolescents: results from the Québec Family Study. Br J Nutr. 2010;104(07):1074-9.

42. Bryant EJ, King NA, Blundell JE. Disinhibition: its effects on appetite and weight regulation. Obes Rev. 2008;9(5):409-19.

43. Zyriax B, Wolf $C$, Schlüter A, Khattak AH, Westenhoefer J, Windler E. Association of cognitive dietary restraint and disinhibition with prediabetes - cross-sectional and longitudinal data of a feasibility study in German employees. Public Health Nutr. 2012;15(05):860-7.

44. Rossiter E, Wilson G, Goldstein L. Bulimia nervosa and dietary restraint Behav Res Ther. 1989;27(4):465-8.
45. Schur EA, Heckbert SR, Goldberg JH. The association of restrained eating with weight change over time in a community-based sample of twins. Obesity. 2010;18(6):1146-52.

46. Westenhoefer J. Dietary restraint and disinhibition: is restraint a homogeneous construct? Appetite. 1991;16:45-55.

47. Sherain E, Russ M, Hull J, Clarkin J, Smith G. Construct validity of the three-factor eating questionnaire: flexible and rigid control subscales. Int J Eat Disord. 1994;16(2):187-98.

48. Provencher V, Drapeau V, Tremblay A, Despre's J, Lemieux S. Eating behaviors and indexes of body composition in men and women from the Qubec family study. Obes Res. 2003;11(6):783-92

49. Jastreboff AM, Gaiser EC, Gu P, Sinha R. Sex differences in the association between dietary restraint, insulin resistance and obesity. Eat Behav. 2014;15(2):286-90

50. Hays NP, Roberts SB. Aspects of eating behaviors "Disinhibition" and "Restraint" are related to weight gain and BMI in women. Obesity. 2008;16(1):52-8

\section{Submit your next manuscript to BioMed Central and take full advantage of:}

- Convenient online submission

- Thorough peer review

- No space constraints or color figure charges

- Immediate publication on acceptance

- Inclusion in PubMed, CAS, Scopus and Google Scholar

- Research which is freely available for redistribution 